\title{
Transcripts that associate with the RNA binding protein, DEAD-END (DND1), in embryonic stem (ES) cells
}

Rui Zhu', Michelina lacovino ${ }^{2}$, Elisabeth Mahen², Michael Kyba² and Angabin Matin ${ }^{1 *}$

\begin{abstract}
Background: The RNA binding protein, DEAD END (DND1), is essential for maintaining viable germ cells in vertebrates. It is also a testicular germ cell tumor susceptibility factor in mice. DND1 has been shown to interact with the $3^{\prime}$-untranslated region (3'-UTR) of mRNAs such as P27 and LATS2. Binding of DND1 to the 3'-UTRs of these transcripts blocks the inhibitory function of microRNAs (miRNA) from these transcripts and in this way DND1 helps maintain P27 and LATS2 protein expression. We found that DND1 is also expressed in embryonic stem (ES) cells. Because ES cells share similar gene expression patterns as germ cells, we utilized ES cells to identify additional candidate mRNAs that associate with DND1.

Results: ES cells are readily amenable to genetic modification and easier to culture in vitro compared to germ cells. Therefore, for the purpose of our study, we made a genetically modified, stable, human embryonic stem (hES) cell line that expresses hemagluttinin (HA)-tagged DND1 in a doxycycline (dox) regulatable manner. This line expresses modest levels of HA-DND1 and serves as a good system to study DND1 function in vitro. We used this stable cell line to identify the transcripts that physically interact with DND1. By performing ribonucleoprotein immunoprecipitation (RIP) followed by RT-PCR, we identified that transcripts encoding pluripotency factors (OCT4, SOX2, NANOG, LIN28), cell cycle regulators (TP53, LATS2) and apoptotic factors (BCLX, BAX) are specifically associated with the HA-DND1 ribonucleoprotein complex. Surprisingly, in many cases, bioinformatics analysis of the pulleddown transcripts did not reveal the presence of known DND1 interacting motifs.

Conclusions: Our results indicate that the inducible ES cell line system serves as a suitable in vitro system to identify the mRNA targets of DND1. The RIP-RT results hint at the broad spectrum of mRNA targets that interact with DND1 in ES cells. Based on what is known about DND1 function, our results suggest that DND1 may impose another level of translational regulation to modulate expression of critical factors in ES cells.
\end{abstract}

\section{Background}

Inactivation of the Dnd1 gene results in sterility in vertebrates as well as causes development of testicular germ cell tumors in mice [1,2]. Dnd1 function is essential to maintain viable germ cells in vertebrates [3,4]. Mice with inactivated Dnd1 show progressive reduction in germ cell numbers starting around embryonic day (E) 8 and are therefore rendered sterile at birth. In addition, when inactivation of Dnd1 occurs in 129 strain mouse background, these mice have a very high incidence of

\footnotetext{
* Correspondence: amatin@mdanderson.org

'Department of Genetics, University of Texas, MD Anderson Cancer Center, Houston, TX 77030, USA

Full list of author information is available at the end of the article
}

testicular germ cell tumors [5-7]. Thus, on the 129 background, some germ cells escape death to undergo cancerous transformation. The transformed germ cells eventually differentiate randomly into myriad cell types that constitute the teratomas or teratocarcinomas in the testes. The testicular germ cell tumors in mice resemble human pediatric testicular type I germ cell tumors $[8,9]$.

DND1 has canonical RNA recognition motifs (RRM) $[1,10,11]$. Mutations engineered in the RRM prevent interaction of DND1 with mRNAs and have also been reported to prevent nucleo-cytoplasmic translocation of zebrafish DND1 [10]. In addition, a disease associated nucleotide polymorphism in the highly conserved RRM of DND1 was detected in a human patient with germ cell

\section{Biomed Central}


tumor [12]. Previous reports have shown that DND1 interacts with the 3'-untranslated region (UTR) of mRNAs such as that of the cell cycle inhibitor, P27 $\left(\mathrm{p} 27^{\mathrm{Kip} 1}, C D K N 1 B\right)$ and cell cycle regulator and tumor suppressor, LATS2 (large tumor suppressor, homolog 2 of Drosophila - a serine/threonine-protein kinase) $[11,13]$. DND1 interacts with U-rich sequences found in the 3'-UTR of P27. In the case of P27, interaction of DND1 hindered miR-221 access to P27 3'-UTR. This led to increased expression levels of P27. Although DND1 inhibits mir-372 and 373 from LATS2 mRNA, the DND1-binding sequences on the 3'-UTR of LATS2 have not been identified. DND1 was also shown to interact with the U-rich sequences on zebrafish Nanos 1 and TDRD7 mRNAs.

The exact mechanism as to how DND1 prevents miRNA-mediated translation repression is unclear. In the case of P27, Nanos1 and TDRD7, the U-rich sequences are found adjacent to miRNA binding sites $[10,11]$. This suggests that DND1 may bind to mRNA to physically displace the miRNAs and miRISC (miRNA-induced silencing complexes). An alternate possibility is that DND1 may bind mRNA and sequester it away from miRNA access.

In the mouse, DND1 is detected in the early embryo $[2,14]$ and then becomes enriched in primordial germ cells (PGCs) after $\sim$ E8 [3,15]. We also detected DND1 expression in mouse (mES) [14] and human embryonic stem cells (hES). PGCs and ES cells share gene expression patterns, markers and miRNAs in common [16]. For example, both PGCs and ES cells express cellular markers such as OCT4, VASA, NANOG and FRAGILIS. They express similar miRNA families such as miR 209-295, miR 302367 and miR 17-92 [17-20] and express critical pluripotency factors such as OCT4, SOX2 and NANOG [3,21-24]. Indeed, there is speculation that ES cells may be derived from germ cells of the early embryo [25]. Because ES cells express DND1, it is expected that they also normally express the mRNAs and miRNAs whose activity is modulated by DND1. Moreover, ES cells are easily cultured in vitro and are more experimentally amenable compared to germ cells. Therefore, ES cells should serve as a good system to study DND1 function. We therefore sought to determine the mRNA targets of DND1 in hES cells. We expect that there are shared sets of mRNA targets in PGCs and ES cells and knowledge of the nature of the transcripts that associate with DND1 will illuminate the function of DND1 in pluripotent cell types such as in ES and germ cells.

\section{Methods}

\section{Generation of stable hES/HA-DND1 cell lines}

The hES cell lines H1 (WA01) and H9 (WA09) (WiCell Research Institute, Madison, WI) [26] were cultured on mitotically inactivated mouse embryonic fibroblasts or under feeder free conditions on Matrigel (BD Biosciences). H9 (WA09) hES lines were genetically modified to stably express HA-tagged human DND1 in an inducible manner. Two recombinant lentiviral constructs were introduced into $\mathrm{H} 9$ cells. Introduction of the first lentivirus, which expresses transactivator (rtTA) from a constitutive Ubiquitin promoter (UbC), generated $\mathrm{H} 9$ rtTA cells [27]. The second virus encodes the inducible target locus (DND1 with HA-tag cloned at 3' end) downstream of the doxycycline-responsive promoter (sgTRE) [28], and bears an IRES-GFP reporter (Figure 1c). The cell line (hES/HA-DND1 cells) containing both viruses was derived by two rounds of transient and low dose doxycycline induction (50 ng/uL, overnight), followed by flow cytometry for GFP+ cells.

Thus, the derived hES/HA-DND1 cells use the tetracycline on (Tet-On) system, in which treatment of cells with doxycycline (dox) causes dox-activated transactivator to interact with the sgTRE element to turn on expression of HA-DND1.

\section{FACS analysis}

Dual labeling using anti-SSEA-4 and anti-OCT4 antibodies was performed on hES/HA-DND1 cell lines to determine the percent of undifferentiated cells in the population. hES/HA-DND1 cells ranged from 50\% to $77 \%$ positive (double-labeled) for the markers OCT4 and SSEA-4. These hES/HA-DND1 cells were subsequently passaged and treated with dox prior to performing RIP experiments.

\section{Induction of HA-DND1 with increasing concentrations of doxycycline}

hES/HA-DND1 cells, grown on Matrigel in 6-well plates, were treated with increasing concentrations, $100-500 \mathrm{ng} / \mathrm{mL}$, of doxycycline (dox) for $24 \mathrm{~h}$. The cells were lysed and used for immunoblotting.

Immunoblotting with rabbit anti-DND1 antibody, antiHA antibody and anti-GAPDH was performed as described [14]. Densitometric analysis was performed to quantitate the band intensities.

\section{Ribonucleoprotein immunoprecipitation (RIP or RNP immunoprecipitation) assay}

RIP was performed essentially as described in [29]. 3 independent RIP experiments followed by RT-PCR were performed and one representative result is shown (Figures 2 and 3). hES/HA-DND1 cells, grown on Matrigel, were treated with dox $(500 \mathrm{ng} / \mathrm{mL})$ for $24 \mathrm{~h}$. Control ES cells were untreated. The cells were subsequently lysed and cell extracts were made with mild lysis buffers to minimize exchange of mRNAs between proteins. The lysates (also referred to as mRNP extracts) were incubated with antiHA antibody (Sigma) linked to agarose beads. Excess tRNA (250 ug yeast tRNA) was included to block spurious interaction of RNAs with HA-DND1. As another control, we incubated mRNP extracts from dox treated cells with 
a

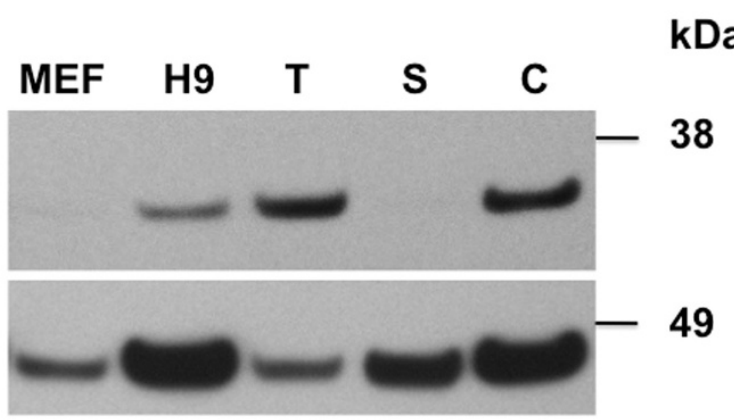

b

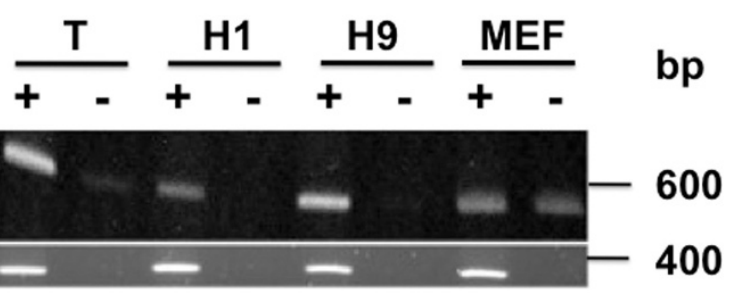

C Lentiviral transactivator expression construct :

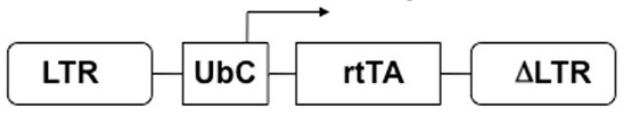

Lentiviral inducible construct :

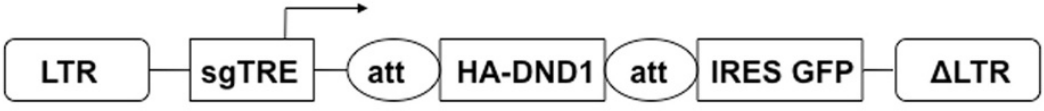

d

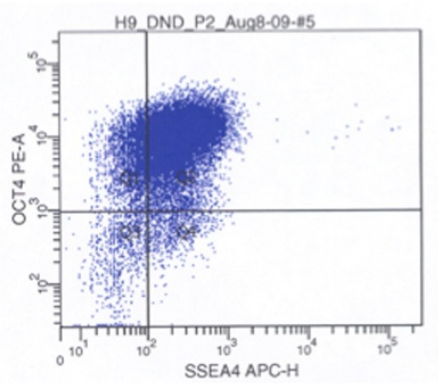

e

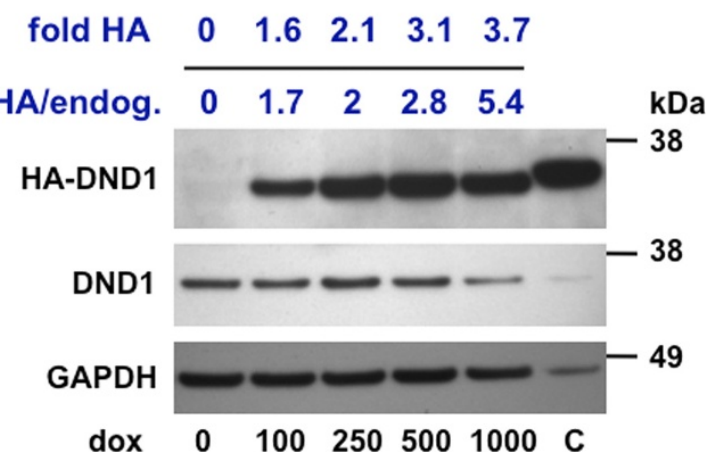

Figure 1 DND1 expression in hES cells. (a) Immunoblotting using anti-DND1 antibody of MEF, hES line H9, mouse testes (T) and spleen (S) (10 $\mathrm{\mu g}$ protein/lane). Control (C) is 293T cells transfected with HA-DND1 encoding expression vector. (bottom) Blot reprobed with anti-GAPDH. (b) RT-PCR to amplify DND1 in human testes (T), hES cells H1, H9, and MEFs. (+) lanes had superscript; (-) had no superscript during RT. (bottom) RT-PCR for HPRT. (c). The first virus expresses rtTA that binds dox. The second virus carries dox-responsive promoter (sgTRE) that drives HADND1 expression. Dox also induces GFP expression, which allows identification of cells carrying both constructs. att (sites for recombinational cloning); LTR and $\triangle$ LTR (long terminal repeats); IRES (internal ribosome entry site). (d) FACS analysis indicates hES/HA-DND1 cells maintain high expression (77\% +ve) of OCT4 and SSEA-4 (quadrant Q2, top right). (e) hES/HA-DND1 cells induced to express HA-DND1 upon dox treatment (100 to $1000 \mathrm{ng} / \mathrm{mL}$ ) for $24 \mathrm{~h}$. Immunoblotting using anti-HA antibody (top panel), anti-DND1 antibody (middle panel) and anti-GAPDH antibody (bottom panel) (20 $\mathrm{mg}$ hES/HA-DND1 lysates/lane). Relative HA-DND1 levels (fold HA) range from 1.6 - 3.7 fold with increasing dox. (Relative HADND1 of each lane is ratio of HA-DND1/GAPDH). The level of endogenous DND1 (DND1/GAPDH) in hES/HA-DND1 cells treated with 0 to 1000 $\mathrm{ng} / \mathrm{mL}$ dox was 1.2, 0.9, 1.1, 1.1 and 0.7, respectively. The level of induced HA-DND1 to endogenous DND1 levels (HA/endog) was estimated to be 1.7 to 5.4 -fold, with increasing dox. 


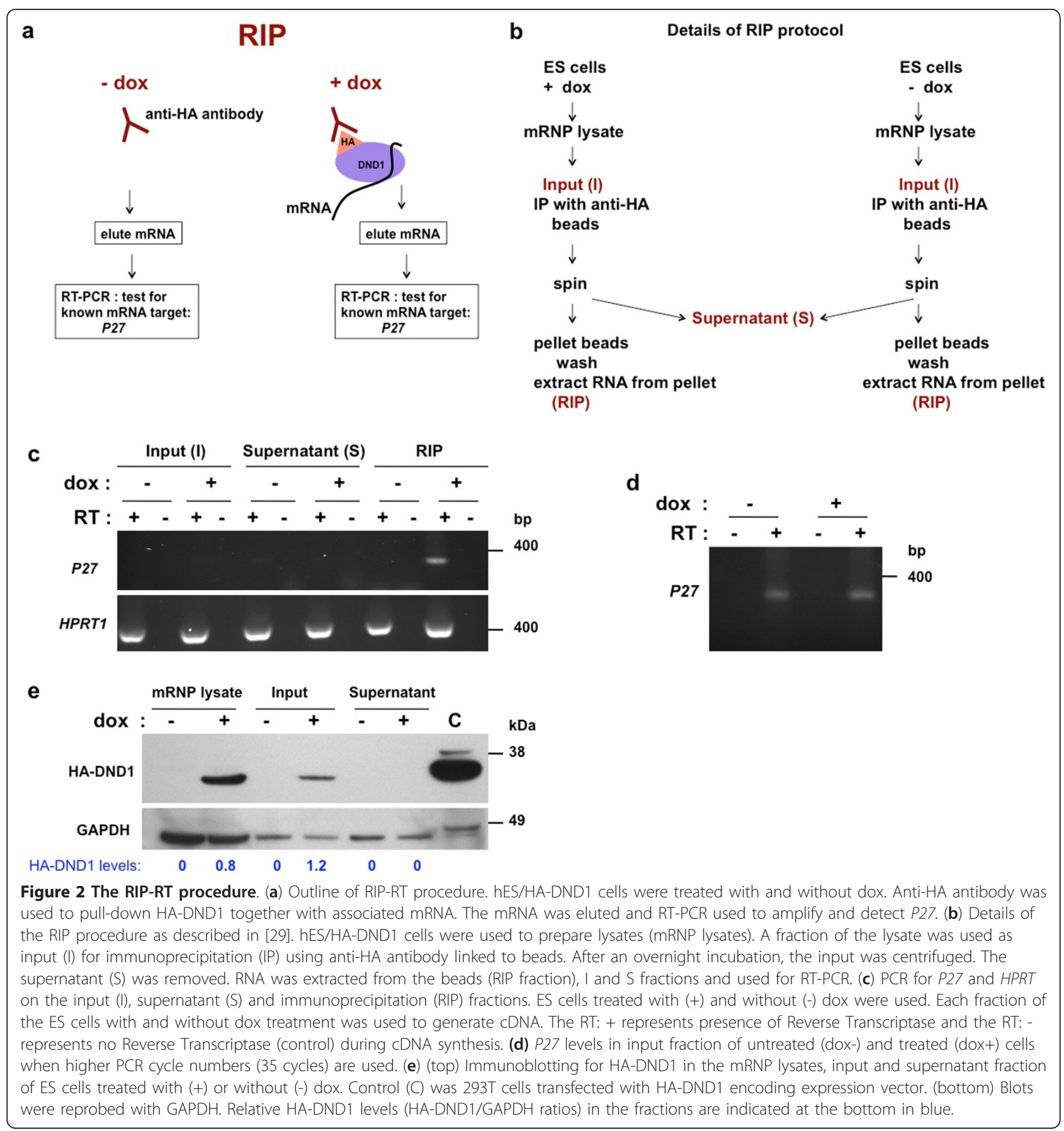

anti-FLAG antibody linked to beads (Sigma). The antiFLAG antibody used was the same IgG1 isotype as the anti-HA antibody.

The beads (linked to anti-HA antibody and now associated with HA-DND1 and RNAs) were pelleted by centrifugation, extensively washed, treated with proteinase $\mathrm{K}$ and the RNA was eluted off. The extracted RNA was used to prepare cDNA. RNA concentrations were measured using NanoDrop spectrophotometer (Thermo
Scientific) and equivalent amounts were used to prepare cDNA. The 'High capacity RNA-to-cDNA Master Mix with NO RT control' kit (applied biosystems) was used for cDNA preparation. cDNA was also prepared from the other fractions: mRNP lysates, input(I) and supernatant (S) (Figure 2b). Aliquots from these fractions were saved during the RIP procedure.

$P C R$ was performed to analyze the RIP, I and S fractions for transcripts encoding P27, HPRT, etc. The primers for 


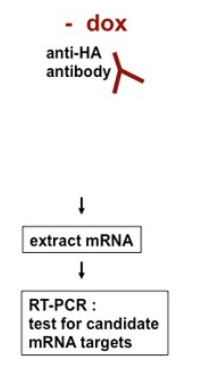

RIP

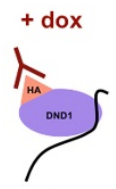

1

extract mRNA

$\downarrow$

RT-PCR :
test for candidate

test for candidate
mRNA targets
+ dox

anti-FLAG

antibody

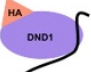

$\downarrow$

extract mRNA

$\downarrow$

RT-PCR :

test for candidate
mRNA targets

b

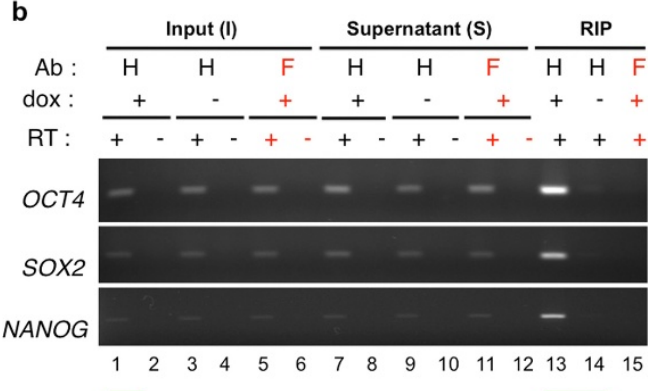

c

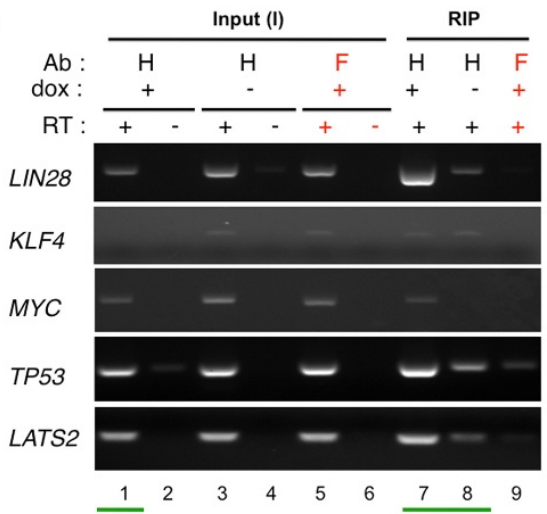

d

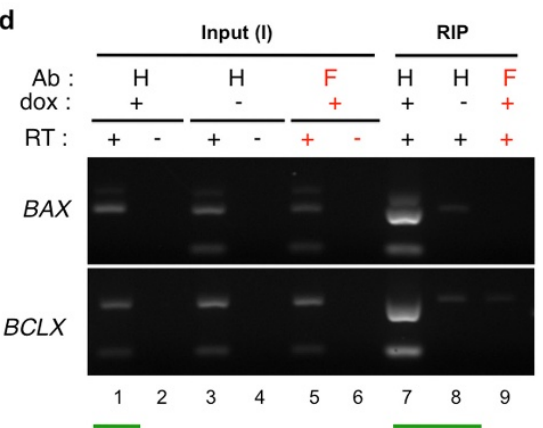

Figure 3 (a) Outline of RIP-RT and controls. hES/HA-DND1 cells were treated with dox (+DOX) or not treated with dox (-DOX). The cells were used to make mRNP lysates and the input fraction was incubated with anti-HA antibody or as control, anti-FLAG antibody for RIP. Excess tRNA was added to the input. RNA extracted from the RIP fraction was used for RT-PCR. (b) Defining the pluripotency factors which are targets of DND1. Anti-HA antibody (a-H) or anti-FLAG (a-F) was used for RIP. After RIP, RT-PCR was performed on equal amounts of cDNA from I, S and RIP fractions. RT+ indicates presence of Reverse Transcriptase during CDNA preparation. RT- are control lanes with no Reverse Transcriptase. Specific pull down of OCT4, SOX2 and NANOG by anti-HA antibody is observed in lane 13. The PCR cycle number used to detect each transcript is described in Methods. (c) Transcripts encoding pluripotency factors and cell cycle regulators targets are targets of DND1. RT-PCR of the S fraction was not performed on these samples. Specific pull down of LIN28, TP53 and LATS2 by anti-HA antibody is observed in lane 7. (d) Pro-apototic factor BAX and anti-apoptotic factor BCLX are specifically pulled down with HA-DND1 during RIP using anti-HA antibodies (lane 7). 
RT-PCR were as follows [30-34]: P27-F ttaaaaatacatatcgctgacttc, P27-R cttcatacccgctccac, OCT4-F gacaacaatgaaaatcttcaggaga, OCT4-R ctggcgccggttacagaacca, SOX2-F ccccctgtggttacctcttc, SOX2-R ttctccccctccagttcg, LATS2-F gcgacctctgggatgatgtg, LATS2-R ttggagtcccaccagtgaa, NANOG-F atgcctgtgatttgtgggcc, NANOG-R gccagttgttttctgccac, MYC-F cccgcgacgatgccctcaac, MYC$\mathrm{R}$ agaagccgctccacatacagtcct, LIN28-F acgtgcgcatggggttcg, LIN28-R tgggccttcagcggacat, KLF4-F aggtggcgggctgatgggcaagtt, KLF4-R gaagcggcagggcagggtgacagt, TP53-F cccc tcctggccctgtcatctt, TP53-R ccgggcgggggtgtggaatcaac, BCLX-F catggcagcagtaaagcaag, BCLX-R gcattgttcccatagag ttcc, BAX-F ggcccaccagctctgagcagatc; BAX-R gacaacccg gcccagttgaagtt, HPRT-F gttgagagatcatctccacc, HPRT-R agctatgatgaaccaggtta.

The PCR cycle for each transcript was adjusted depending on the nature of the primer and abundance of the transcript, as determined in preliminary experiments (data not shown). PCR cycles for each transcript was as follows: P27 (30 or 35 cycles), HPRT1 (35 or 24 cycles), OCT4, SOX2 and NANOG (26 cycles), LIN28, KLF4, MYC, TP53, LATS2, BAX and BCLX (35 cycles). The expected sizes of the RT-PCR products are: $216 \mathrm{bp}$ for OCT4, 232 bp for SOX2, 403 bp for NANOG, 394 bp for LIN28, 424 bp for MYC, 347 bp for KLF4, 126 bp for $L A T S 2,199$ bp for TP53, 351 bp xL product from BCLX and 299 bp for $B A X$.

\section{DND1 interacting sequences}

We examined full-length transcripts for the known human P27 DND1 interacting sequence [11] TTTTTCCTTATTT or TTTTTACCTTTT; Nos1 DND1 interacting sequence TGTTTGATTTT or TTTTATTTT and TDRD7 DND1 interacting sequence TTGATTTT. In cases where multiple transcripts are present, all known transcripts were examined for the presence of any of the known DND1 interacting sequences.

\section{Results}

\section{DND1 expression in human ES cells}

We previously detected DND1 expression in G4 mouse ES cells [14]. Using immunoblotting and RT-PCR techniques, we were also able to detect DND1 expression in human embryonic stem cells (hES), H1 (WA01) and H9 (WA09) (Figure 1a and 1b). However, DND1 is not detected in mouse embryo fibroblast (MEF) cells, the feeder cells on which the hES cells grow. In the case of MEFs, bands are observed in both superscript (-) and superscript (+) lanes (Figure 1b). These likely are nonspecific bands and indicate no specific DND1 expression in MEFs, as was also previously shown [14].

Doxycycline-inducible expression of HA-DND1 in hES cells We established lentiviral modified hES lines that express HA-tagged human DND1 (hES/HA-DND1) in an inducible manner. The hES/HA-DND1 cell line uses the tetracycline on (Tet-On) system in which doxycycline turns on the expression of HA-DND1 [35,36] (Figure 1c). The hES/HA-DND1 cell line showed a modest dosedependent increase of HA-DND1 levels (1.6 to 3.7 - fold increase of HA-DND1 levels) upon treatment with increasing concentrations of dox for $24 \mathrm{~h}$ (Figure 1e, top panel). We also determined the levels of endogenous DND1 expression using anti-DND1 antibody (Figure 1e, middle panel). The level of endogenous DND1 in hES/ HA-DND1 cells treated with increasing dox was fairly similar (band intensities were 1.2, 0.9, 1.1, 1.1 and 0.7 with 0 to $1000 \mathrm{ng} / \mathrm{mL}$ dox, respectively). Comparing the levels of induced HA-DND1 to endogenous DND1 (HA/endog, Figure 1e), we estimate that HA-DND1 levels range from about a 2 to 6 -fold increase compared to endogenous DND1. For subsequent RIP experiments, we treated hES/ HA-DND1 cells with $500 \mathrm{ng} / \mathrm{mL}$ dox for $24 \mathrm{~h}$ which induces a 2.8-fold higher level of HA-DND1 compared to endogenous DND1 levels.

\section{HA-DND1 associated P27 mRNA}

First, we performed RIP $[29,37,38]$ to test whether HAtagged DND1, induced to be expressed in the hES/HADND1 cell line, can pull down $P 27$ mRNA. This is because it has been previously shown that DND1 binds to the 3'-UTR of P27 mRNA [11].

hES/HA-DND1 cells were treated with dox $(500 \mathrm{ng} / \mathrm{mL}$ for $24 \mathrm{~h}$ ) whereas control cells were untreated. The cells were subsequently lysed to make the cell extracts that were used to perform RIP as described in Methods [29] and outlined in Figure 2.

We used RT-PCR to analyze the RIP, I and S fractions for P27. cDNA was made using equal amounts $(25 \mathrm{ng})$ of RNA from each fraction (input, supernatant and RIP; +/dox treated fractions). Equivalent amounts of cDNA were then used for RT-PCR. Our results showed that P27 levels were barely detectable in the input fraction (I) but enriched in the RIP fraction (Figure 2c). This indicates that P27 mRNA is indeed pulled down specifically with HA-DND1 in the RIP fraction (Figure 2c). Although P27 is barely detectable in the input lanes in Figure 2c, P27 is normally present in the hES/HA-DND1 cells as it can be detected in both untreated and dox-treated cells using higher PCR cycles (Figure 2d).

In contrast, RT-PCR for HPRT indicated that it is present in all the fractions in dox treated and untreated cells. This rules out specific interaction of HPRT mRNA with HA-DND1.

In addition, we monitored for the presence of HADND1 in the different fractions. Immunoblotting using anti-DND1 show that the HA-DND1 was present in the dox treated ES cells and in the input (I) fraction, but not in the supernatant (S) fraction (Figure 2e). This 
indicates that the majority of induced HA-DND1 in the input (I) fraction was efficiently immunoprecipitated by anti-HA antibody and pelleted in the RIP fraction. Because of proteinase $\mathrm{K}$ digestion prior to RNA elution, immunoblotting could not be performed on the RIP fraction.

\section{Pluripotency factors, tumor suppressors and apoptotic factors are DND1 targets}

Next, we examined whether transcripts of pluripotency factors associate with DND1. This is because DND1 is normally expressed in both ES cells and primordial germ cells (PGCs) and both of these cell types express pluripotency factors. Thus, we hypothesized that transcripts encoding pluripotency factors may be targets of DND1 in ES cells. Therefore, we performed RT-PCR on RIP fractions to test for pull-down of mRNAs of candidate pluripotency factors such as OCT4, SOX2, NANOG, LIN28, $K L F 4$ and $C M Y C$. We also examined whether the mRNA transcripts of cell cycle regulators and tumor suppressors (LATS2, TP53) and apoptotic factors $(B A X, B C L X)$ are targets of DND1.

The results from RIP-RT experiments showed that the following pluripotency factors are specific targets of DND1: OCT4, SOX2, NANOG and LIN28 (Figure 3b and 3c). In the case of OCT4, SOX2, NANOG (Figure 3b) we observe specific pull down of the transcripts in lane 13 (anti-HA, dox + , RT + ) compared to lane 14 (anti-HA, dox -, RT +). In addition we observed enrichment of transcript compared to that in the Input (I) lane (lane 1). In contrast, similar comparison of the transcript levels in the test and control lanes with the input lanes (Figure 3c, lanes 7, 8 and 1), lead us to conclude that KLF4 and C $M Y C$ are not specific targets of DND1. We note that faint bands are observed in the control RIP lanes (Figure 3c, d, lanes 8 and 9). These bands indicate that anti-HA antibody (in -dox cells, lane 8) and anti-FLAG antibody (in +dox cells, lane 9) can also pull down low levels of transcripts, which represent non-specific interactions. In comparison, specific pull-down of transcripts occurs at higher levels (using anti-HA antibody in +dox cells, lane 7).

TP53 and LATS2 are also specifically pulled down in the RIP fraction of dox-treated cells (Figure 3c, comparing lanes 7 and 8). However, in the case of TP53 and LATS2, there was not significant enrichment in the RIP fraction (Figure 3c, lane 7) compared to the input (I) lane (Figure 3c, lane 1). This indicates that LIN28 is more efficiently pulled down with DND1 compared to TP53 and LATS2.

On the other hand, transcripts of both the pro-apoptotic factor $B A X$ and the anti-apoptotic factor $B C L X$ are very efficiently pulled down with DND1 (Figure $3 \mathrm{~d}$ ). There is also significant enrichment of $B A X$ and $B C L X$ transcripts in the RIP fraction of dox treated cells (Figure $3 \mathrm{~d}$, comparing lanes 7 to 8 and 1 ).

\section{DND1 binding sequences in the $3^{\prime}$-UTRs of mRNA transcripts}

Next, we examined whether the transcripts studied contains the known DND1 binding sequences. We therefore examined the full-length transcripts for U-rich DND1 interacting sequences that were previously mapped in the 3'-UTR of P27 [11] as well as for sequences that were mapped to the 3'-UTR of Nanos1 and TDRD7. In cases where multiple transcripts of a gene are known, all the transcripts were examined for the presence of any of the DND1 interacting sequence. We found that none of the transcripts that we examined in this study contained the same DND1 interacting sequence as P27. However, the 3'-UTRs of OCT4, NANOG, MYC and LIN28 harbored sequences similar to the DND1 interacting sequences found in TDRD7. LATS2 3'-UTRs contained the DND1 interacting sequences similar to that found in Nanos 1 (Table 1).

Thus, sequence examination of transcripts alone does not predict whether a particular transcript will bind to and be pulled-down with DND1. Although $M Y C$ did not bind to HA-DND1, the 3'-UTR of one of its transcripts contains putative DND1 binding sequences. In contrast, even though $B A X$ and $B C L X$ bound very efficiently to DND1 ribonucleoprotein complex, we did not find DND1 binding sites in any of its transcripts. This suggests that there likely exists additional DND1 binding sequences that are yet to be defined. To test this, we aligned the 3'-UTRs of the DND1 interacting mRNAs to examine for any common sequences. We found some regions with weak consensus that may represent novel DND1 binding sites. Further experimental analysis will be required to establish whether these are indeed DND1 binding sites.

The other possibility is that interaction of mRNAs with DND1 may be mediated through co-interaction of DND1 with other proteins and thus presence of DND1 binding sequences within mRNAs may not be essential for interaction.

\section{Discussion}

We demonstrate use of a stable, inducible ES cell line to identify transcripts that associate with DND1. Our results show that transcripts of pluripotency factors associate with DND1. OCT4, SOX2 and NANOG are key transcription factors essential for maintenance of the pluripotent state of ES cells [39-41] and are also important for early germ cells $[3,22,24]$. These results lead us to hypothesize that DND1 likely imposes another level of regulation of expression of pluripotency factors. However, it remains to be determined how 
Table 1 Transcripts with potential DND1 binding sequences

\begin{tabular}{|c|c|c|c|}
\hline Gene & Number of transcripts ${ }^{1}$ & DND1 interacting sequence ${ }^{2}$ & Interaction with DND1 ${ }^{3}$ \\
\hline P27 (CDKN1B) & 4 & $\begin{array}{l}\text { CDKN1B-001 (ENST00000228872) } \\
\text { 1196- TTITCCTATTT -1208 } \\
\text { 1236- TTITACCTIT -1247 } \\
\text { CDKN1B-002 (ENST00000442489) } \\
\text { 537- TITTCCTATTT -549 }\end{array}$ & + \\
\hline HPRT1 & 3 & - & - \\
\hline OCT4 (POU5F1) & 6 & $\begin{array}{l}\text { POU5F1-001 (ENST00000434616) } \\
\text { 1345- TTGATTIT -1352 } \\
\text { POU5F1-002 (ENST00000412166) } \\
\text { 1088- TTGATTT -1095 } \\
\text { POU5F1-004 (ENST00000463773) } \\
621-\text { TTGATTIT -628 }\end{array}$ & + \\
\hline SOX2 & 1 & - & + \\
\hline NANOG & 3 & $\begin{array}{l}\text { NANOG-001 (ENST00000229307) } \\
\text { 2042- TTGATTIT -2049 }\end{array}$ & + \\
\hline LIN28 & 2 & $\begin{array}{l}\text { LIN28A-001 (ENST00000326279) } \\
\text { 3879- TTGATTT -3886 } \\
\text { LIN28A-201 (ENST00000254231) } \\
\text { 3326- TTGATTIT -3333 }\end{array}$ & + \\
\hline KLF4 & 7 & - & - \\
\hline$\overline{M Y C}$ & 5 & $\begin{array}{l}\text { MYC-001 (ENST00000377970) } \\
\text { 2283-TTGATTT -2290 }\end{array}$ & - \\
\hline TP53 & 15 & - & + \\
\hline LATS2 & 3 & $\begin{array}{l}\text { LATS2-001 (ENST00000382592) } \\
4176-\text { TTTATTT }-4184\end{array}$ & + \\
\hline$\overline{B A X}$ & 12 & - & + \\
\hline$\overline{B C L X(B C L 2 L 1)}$ & 10 & - & + \\
\hline
\end{tabular}

DND1 association with these transcripts affect ES cell properties.

miRNAs target multiple mRNAs simultaneously. On the other hand, DND1 is also able to inhibit miRNA activity from multiple mRNAs [11,13]. Our results also hint at the broad range of mRNA targets of DND1. Further studies are needed to elucidate the role of DND1 in modulating specific miRNA activity from each mRNA.

Our RIP-RT results show that the transcripts of the cell cycle regulators, TP53 and LATS2 mRNA are also targets of DND1. This observation is corroborated by a recent study where RIP technique was applied to NIH3T3 cells and showed that tagged DND1 is able to pull down a number of cell cycle regulators including Lats 2 and $p 53$ [42].

In addition, mRNA transcripts of both the pro-apoptotic factor $B A X$ and the anti-apoptotic factor mRNA, BCLX, are efficiently pulled down with DND1. Thus DND1 interacts with a broad variety of targets and with mRNAs that have opposite effects, as for example, DND1 associates with mRNAs of both anti- and pro-apoptotic factor, $B C L X$ and $B A X$, respectively. This raises the question as to what confers specificity for DND1 function? One possibility is that there exist factors that favor interaction with specific mRNAs at particular stages of the cell cycle or under certain physiological conditions. It is also likely that intrinsic and cellular factors may regulate DND1 function to promote cell survival and maintain a pluripotent cell state. Depending on the physiological state of the cell, the balance of expression of the opposing factors likely determine cell fate, death or transformation.

Germ cells, testicular cancer cells and ES cells share gene expression patterns $[21,24,43]$. For example, SOX2, NANOG and LIN28 expression can be used to distinguish different histological subtypes of human testicular cancers $[44,45]$. This is thought to reflect the origin of testicular cancer from germ cells. Whether the same pluripotency factors also associate with DND1 in germ cells will have to be experimentally determined. Interestingly, our observation that DND1 interacts with pluripotency and anti-apoptotic factor mRNAs may explain why lack of DND1 results in the death of germ cells in mice. Pluripotency factors are able to reprogram differentiated adult cell types into iPS (induced pluripotent 
stem) cells. For example, human iPS cells require OCT4, SOX2, NANOG, and LIN28 whereas mouse iPS cells require Oct4, Sox2, Myc and Klf4 [46,47]. Interestingly, we found that transcripts encoding OCT4, SOX2, NANOG and LIN28 but not KLF4 associate with DND1 in hES cells.

Surprisingly, we found that DND1 interacting sequence similar to $P 27$ are rarely present in other transcripts. However, the 3'-UTR of some transcripts harboured DND1 interacting sequences described in Nanos1 and TDRD7. Therefore, at present, based on sequence analysis alone, it is difficult to predict whether a particular transcript interacts with DND1.

\section{Conclusion}

In conclusion, we report the generation of the inducible hES cell system (hES/HA-DND1 cells) and demonstrate the advantage of using this stable, dox inducible hES cell system to identify the RNA targets of DND1. hES cells closely resemble primordial germ cells and we can induce and control expression of modest levels of HA-tagged DND1. Using the hES/HA-DND1 cells, we demonstrate that transcripts of pluripotency factors, cell cycle regulators and apoptotic factors are associated with DND1 and are likely targets of regulation by DND1. We found that RIP followed by RT-PCR allows us to quickly and readily discriminate between specific and non-specific mRNA targets of DND1. Further techniques such as microarray or RNA-Seq following RIP will be useful to identify the global targets of DND1 from ES cells. Although a number of transcripts associate with DND1, surprisingly, DND1 interacting sequence similar to $P 27$ are rarely present in other transcripts. Thus additional DND1 interacting sequences remain to be identified.

Our result suggests that DND1 imposes another level of translational regulation that may modulate expression of critical factors in ES cells. Because DND1 is implicated in reciprocally regulating miRNA mediated translation suppression of specific target mRNAs, this suggests that the balance between DND1 and miRNAs modulates context-dependent mRNA targets.

\footnotetext{
Abbreviations

DND1: (dead end homolog 1 (zebrafish)); HA-DND1: (hemagluttinin-tagged DND1); P27 (CDKN1B: cyclin-dependent kinase inhibitor 1B, Kip1); LATS2: (large tumor suppressor, homolog 2 of Drosophila); OCT4 (POU5F1: POU class 5 homeobox 1); SOX2: (SRY (sex determining region Y)-box 2); NANOG: (Nanog homeobox); LIN28: (lin-28 homolog A (C. elegans)); KLF4: (Kruppellike factor 4); CMYC: (v-myc myelocytomatosis viral oncogene homolog (avian)); TP53: (tumor protein p53); BCLX (BCL2L1: BCL2-like 1); BAX: (BCL2associated $\times$ protein); HPRT: (hypoxanthine phosphoribosyltransferase 1); GAPDH: (glyceraldehyde-3-phosphate dehydrogenase); dox: (doxycycline); RIP: (ribonucleoprotein immunoprecipitation)
}

\section{Acknowledgements}

We thank R. J. Milczareck and A. Crane for technical help in culturing hES cells. We thank K. Luo for technical help and E. Goodell, T. Zwaka, R.
Behringer and J. Deng for invaluable suggestions and discussion. This work was supported by GM081627 to MK and AM and Genes \& Development Hearst Foundation Innovative Research and Educational Development Award to AM.

\section{Author details}

${ }^{1}$ Department of Genetics, University of Texas, MD Anderson Cancer Center, Houston, TX 77030, USA. Pediatric Hematology-Oncology and Blood and Marrow Transplantation Program, University of Minnesota, Minneapolis, MN 55455, USA.

\section{Authors' contributions}

RZ characterized the hES/HA-DND1 cells, performed the RIP experiments and participated in writing of the manuscript. MK conceived the design and generation of hES/HA-DND1 cells. MI, EM and MK generated the hES/HADND1 cells.

AM conceived the study, participated in its design and coordination and wrote the manuscript. All authors read and approved the final manuscript.

Received: 5 May 2011 Accepted: 18 August 2011

Published: 18 August 2011

\section{References}

1. Weidinger G, Stebler J, Slanchev K, Dumstrei K, Wise C, Lovell-Badge R, Thisse C, Thisse B, Raz E: dead end, a novel vertebrate germ plasm component, is required for zebrafish primordial germ cell migration and survival. Curr Biol 2003, 13(16):1429-1434.

2. Youngren KK, Coveney D, Peng X, Bhattacharya C, Schmidt LS, Nickerson ML, Lamb BT, Deng JM, Behringer RR, Capel B, et al: The Ter mutation in the dead end gene causes germ cell loss and testicular germ cell tumours. Nature 2005, 435:360-364.

3. Hayashi K, de Sousa Lopes SMC, Surani MA: Germ cell specification in mice. Science 2007, 314:394-396.

4. Saga Y: Mouse germ cell development during embryogenesis. Curr Opin Genet Dev 2008, 18:337-341.

5. Noguchi T, Stevens LC: Primordial germ cell proliferation in fetal testes in mouse strains with high and low incidences of congenital testicular teratomas. J Natl Cancer Inst 1982, 69(4):907-913.

6. Noguchi T, Noguchi M: A recessive mutation (ter) causing germ cell deficiency and a high incidence of congenital testicular teratomas in 129/Sv-ter mice. J Natl Cancer Inst 1985, 75(2):385-392.

7. Stevens LC: A new inbred subline of mice (129-terSv) with a high incidence of spontaneous congenital testicular teratomas. $J$ Nat/ Cancer Inst 1973, 50(1):235-242.

8. Oosterhuis JW, Looijenga LHJ: Testicular germ-cell tumours in a broader perspective. Nature Rev Cancer 2005, 5:210-222.

9. Rescorla FJ: Pediatric germ cell tumors. Semin Surgical Oncology 1999, 16:144-158.

10. Slanchev K, Stebler J, Goudarzi M, Cojocaru V, Weidinger G, Raz E: Control of dead end localization and activity - implications for the function of the protein in antagonizing miRNA function. Mech Dev 2009, 126:270-277

11. Kedde M, Strasser MJ, Boldajipour B, Vrielink JA, Slanchev K, le Sage C, Nagel R, Voorhoeve PM, van Duijse J, Orom UA, et al: RNA-Binding Protein Dnd1 Inhibits MicroRNA Access to Target mRNA. Cell 2007, 131:1273-1286.

12. Linger $R$, Dudakia $D$, Huddart $R$, Tucker $K$, Friedlander M, Phillips KA, Hogg D, Jewett MA, Lohynska R, Daugaard G, et al: Analysis of the DND1 gene in men with sporadic and familial testicular germ cell tumors. Genes Chromosomes Cancer 2008, 47:247-252.

13. Ketting RF: A Dead End for MicroRNAs. Cell 2007, 131:1226-1227.

14. Bhattacharya C, Aggarwal S, Zhu R, Kumar M, Zhao M, Meistrich ML, Matin A: The mouse dead-end gene isoform alpha is necessary for germ cell and embryonic viability. Biochem Biophys Res Commun 2007, 355:194-199.

15. Yabuta $Y$, Kurimoto K, Ohinata $Y$, Seki $Y$, Saitou M: Gene expression dynamics during germline specification in mice identified by quantitative single-cell gene expression profiling. Biol Reprod 2006, 75:705-716.

16. Dyce PW, Toms D, Li J: Stem cells and germ cells: microRNA and gene expression signatures. Histol Histopathol 2010, 25(4):505-513. 
17. Chen C, Ridzon D, Lee CT, Blake J, Sun Y, Strauss WM: Defining embryonic stem cell identity using differentition related microRNAs and their potential targets. Mamm Genome 2007, 18:316-327.

18. Houbaviy HB, Murray MF, Sharp PA: Embryonic stem cell-specific microRNAs. Dev Cell 2003, 5:351-358.

19. Houbaviy HB, Dennis $L$, Jaenisch $R$, Sharp PA: Characterization of a highly variable eutherian microRNA gene. RNA 2005, 11:1245-1257.

20. Wang $Y$, Baskerville S, Shenoy A, Babiarz JE, Baehner L, Blelloch R: Embryonic stem cell-specific microRNAs regulate the G1-S transition and promote rapid proliferation. Nat Genet 2008, 40:1478-1483.

21. Almstrup K, Hoei-Hansen CE, Wirkner U, Blake J, Schwager C, Ansorge W, Nielsen JE, Skakkebaek NE, Rajpert-De Meyts E, Leffers H: Embryonic stem cell-like features of testicular carcinoma in situ revealed by genomewide gene expression profiling. Cancer Res 2004, 64:4736-4743.

22. Elliott AM, de Miguel MP, Rebel VI, Donovan PJ: Identifying genes differentially expressed between PGCs and ES cells reveals a role for CREB-binding protein in germ cell survival. Dev Biol 2007, 311:347-358.

23. Clark AT: The Stem Cell Identity of Testicular Cancer. Stem Cell Rev 2007, 3:49-59.

24. Sperger JM, Chen X, Draper JS, Antosiewicz JE, Chon CH, Jones SB, Brooks JD, Andrews PW, Brown PO, Thomson JA: Gene expression patterns in human embryonic stem cells and human pluripotent germ cell tumors. Proc Natl Acad Sci USA 2003, 100:13350-13355.

25. Zwaka TP, Thomson JA: A germ cell origin of embryonic stem cells? Development 2005, 132:227-233.

26. Liu Y, Shin S, Zeng X, Zhan M, Gonzalez R, Mueller F-J, Schwartz CM, Xue H, $\mathrm{Li} \mathrm{H}$, Baker SC, et al: Genome wide profiling of human embryonic stem cells (hESCs), their derivatives and embryonal carcinoma cells to develop base profiles of U.S. Federal government approved hESC lines. BMC Developmental Biology 2006, 6:20.

27. Bosnakovski D, Xu Z, Gang EJ, Galindo CL, Liu M, Simsek T, Garner HR, Agha-Mohammadi S, Tassin A, Coppée F, et al: An isogenetic myoblast expression screen identifies DUX4-mediated FSHD-associated molecular pathologies. EMBO J 2008, 27:2766-2779.

28. Agha-Mohammadi S, O'Malley M, Etemad A, Wang Z, Xiao X, Lotze MT: Second-generation tetracycline-regulatable promoter: repositioned tet operator elements optimize transactivator synergy while shorter minimal promoter offers tight basal leakiness. J Gene Med 2004, 6:817-828.

29. Keene JD, Komisarow JM, Friedersdorf MB: RIP-Chip: the isolation and identification of mRNAs, microRNAs and protein components of ribonucleoprotein complexes from cell extracts. Nature Protocols 2006, 1(1):302-307.

30. Tai M-H, Chang C-C, Olson LK, Trosko JE: Oct4 expression in adult human stem cells: evidence in support of the stem cell theory of carcinogenesis. Carcinogenesis 2005, 26:495-502.

31. Bourguignon LYW, Peyrollier K, Xia W, Gilad E: Hyaluronan-CD44 Interaction Activates Stem Cell Marker Nanog, Stat-3-mediated MDR1 Gene Expression, and Ankyrin-regulated Multidrug Efflux in Breast and Ovarian Tumor Cells. J Biol Chem 2008, 283:17635-17651.

32. Park ET, Gum JR, Kakar S, Kwon SW, Deng G, Kim YS: Aberrant expression of SOX2 upregulates MUC5AC gastric foveolar mucin in mucinous cancers of the colorectum and related lesions. Int J Cancer 2008, $122: 1253-1260$

33. Voorhoeve PM, le Sage C, Schrier M, Gillis AJM, Stoop H, Nagel R, Liu Y-P, van Duijse J, Drost J, Griekspoor A, et al: A genetic screen implicates miRNA-372 and miRNA-373 as oncogenes in testicular germ cell tumors. Cell 2006, 124:1169-1181.

34. Li CY, Chu JY, Yu JK, Huang XQ, Liu XJ, Shi L, Che YC, Xie JY: Regulation of alternative splicing of Bcl-x by IL-6, GM-CSF and TPA. Cell Research 2004, 14:473-479.

35. Ting DT, Kyba M, Daley GQ: Inducible transgene expression in mouse stem cells. Methods Mol Med 2005, 105:23-46.

36. Kyba M, Perlingeiro RCR, Daley GQ: HoxB4 confers definitive lymphoidmyeloid engraftment potential on embryonic stem cell and yolk sac hematopoietic progenitors. Cell 2002, 109:29-37.

37. Townley-Tilson WHD, Pendergrass SA, Marzluff W, Whitfield ML: Genomewide analysis of mRNAs bound to the histone stem-loop binding protein. RNA 2006, 12:1853-1867.
38. Baroni TE, Chittur SV, George AD, Tenenbaum SA: Advances in RIP-chip analysis: RNA-binding protein immunoprecipitation-microarray profiling. Methods Mol Biol 2008, 419:93-108.

39. Chambers I, Smith A: Self-renewal of teratocarcinoma and embryonic stem cells. Oncogene 2004, 23:7050-7160.

40. Niwa H: How is pluripotency determined and maintained? Development 2007, 134:635-646

41. Silva J, Smith A: Capturing pluripotency. Cell 2008, 132:532-536.

42. Cook MS, Munger SC, Nadeau JH, Capel B: Regulation of male germ cell cycle arrest and differentiation by DND1 is modulated by genetic background. Development 2011.

43. Almstrup K, Leffers H, Lothe RA, Skakkebaek NE, Sonne SB, Nielsen JE, Rajpert-De Meyts E, Skotheim Rl: Improved gene expression signature of testicular carcinoma in situ. Int J Andrology 2007, 30:292-303.

44. Emerson RE, Ulbright TM: Intratubular germ cell neoplasia of the testis and its associated cancers: the use of novel biomarkers. Pathology 2010, 42:344-355.

45. Sonne SB, Perrett RM, Nielsen JE, Baxter MA, Kristensen DM, Leffers $H$, Hanley NA, Rajpert-De Meyts E: Analysis of SOX2 expression in developing human testis and germ cell neoplasia. Int J Dev Biol 2010, 54:755-760.

46. Kashyap V, Rezende NC, Scotland KB, Shaffer SM, Persson JL, Gudas LJ, Mongan NP: Regulation of stem cell pluripotency and differentiation involves a mutual regulatory circuit of the Nanog, OCT4, and SOX2 pluripotency transcription factors with polycomb repressive complexes and stem cell microRNAs. Stem Cells Dev 2009, 18:1093-1108.

47. Boheler KR: Stem cell pluripotency: a cellular trait that depends on transcription factors, chromatin state and a checkpoint deficient cell cycle. J Cell Physiol 2009, 221:10-17.

doi:10.1186/1471-2199-12-37

Cite this article as: Zhu et al:: Transcripts that associate with the RNA binding protein, DEAD-END (DND1), in embryonic stem (ES) cells. BMC Molecular Biology 2011 12:37.

\section{Submit your next manuscript to BioMed Central and take full advantage of:}

- Convenient online submission

- Thorough peer review

- No space constraints or color figure charges

- Immediate publication on acceptance

- Inclusion in PubMed, CAS, Scopus and Google Scholar

- Research which is freely available for redistribution

Submit your manuscript at www.biomedcentral.com/submit
C Biomed Central 